\title{
A Parametric Model of Shoulder Articulation for Virtual Assessment of Space Suit Fit
}

\author{
K. Han KIM ${ }^{1}$, Karen S. YOUNG ${ }^{* 1}$, Yaritza BERNAL ${ }^{2}$, Abhishektha BOPPANA ${ }^{3}$, \\ Linh Q. VU ${ }^{2}$, Elizabeth A. BENSON ${ }^{4}$, Sarah JARVIS ${ }^{4}$, Sudhakar L. RAJULU ${ }^{5}$ \\ ${ }^{1}$ Leidos, Inc., Houston, TX, USA; ${ }^{2}$ Geologics Corp., Houston, TX, USA; \\ ${ }^{3}$ NSBRI, Houston, TX, USA; ${ }^{4}$ MEI Technologies, Inc., TX, USA; \\ ${ }^{5}$ NASA Johnson Space Center, TX, USA \\ DOI: $10.15221 / 16.201 \quad$ http://dx.doi.org/10.15221/16.201
}

\begin{abstract}
Suboptimal suit fit is a known risk factor for crewmember shoulder injury. Suit fit assessment is however prohibitively time consuming and cannot be generalized across wide variations of body shapes and poses. In this work, we have developed a new design tool based on the statistical analysis of body-shape scans. This tool is aimed at predicting the skin deformation and shape variations for any body size and shoulder pose for a target population. This new process, when incorporated with CAD software, will enable virtual suit fit assessments, predictively quantifying the contact volume, and clearance between the suit and body surface at reduced time and cost.
\end{abstract}

Keywords: Anthropometry, Digital Human Modeling, Reposing, Ergonomics

\section{Introduction}

It is well known that the human shoulder will deform considerably during motions due to soft tissue deformation (muscle bunching) and clavicle/scapula movement. Restricting shoulder elevation and protraction during shoulder movements can cause increased strain on the muscles around the shoulder, especially the rotator cuff, potentially increasing the risk of injury. Shoulder injury is one of the risks that have the potential to impair crewmembers' performance and health in long-duration spaceflight. Overall, $64 \%$ of crewmembers experience shoulder pain after extravehicular training in a space suit and $14 \%$ of symptomatic crewmembers require surgical repair [1].

Suboptimal suit fit, in particular at the shoulders, has been identified as one of the predominant risk factors. However, traditional suit fit assessments and laser scans represent only a single person's static data, and thus may not be generalized across wide variations of body shapes and poses. Computational models have been developed to estimate human body shapes using different anthropometry parameters [2]-[4], but overall lack in the quantification of skin deformation patterns, which are crucial for suit-to-body contact assessments.

A previous study at NASA used the static poses from subjects to develop a set of quasi-static models of the shoulder joint in different phases of motion. These models represent actual shoulder morphology in a series of postures [5]. They were developed to be used in a CAD environment to determine human-hardware interactions with a new prototype space suit and to give suit designers a representation of how the human shoulder moves when uninhibited by a suit. This method was limited due to the 'assembly' nature of CAD and lack of ability to alter body poses or sizes.

The aim of this work was to develop a software tool based on statistical analysis of a large dataset of crewmembers' body shapes. This tool can predict the skin deformation and shape variations for any body size and shoulder pose for a target population, from which the geometry can be exported and evaluated against suit models in commercial CAD software.

\section{Methods}

\subsection{Body Scanning}

\subsubsection{Subjects}

Twelve subjects ( 9 males and 3 females) participated in body scanning. The average stature and body weight of the subjects were 167.5 (SD 9.8) cm and 74.8 (SD 13.5) $\mathrm{kg}$, which correspond to a body mass index (BMI) 24.1 (SD 2.6) $\mathrm{kg} / \mathrm{m}^{2}$. The mean age was 25.9 (SD 5.5) years. None of the subjects had any known musculoskeletal injuries or pain at the time of testing. 


\subsubsection{Equipment}

A 3dMD optical whole-body scanner (Atlanta, GA, USA) was used, which was set up to scan the subject's whole body geometry within a capture volume of 440 (length) $\times 346$ (width) $\times 225$ (height) $\mathrm{cm}$.

The scanner used 12 sets of camera modules that can capture both the 3-D geometry and color texture of the body.

\subsubsection{Procedures}

The stature and body weight of the subject were measured using an anthropometer and a scale. Male subjects were asked to remove their shirts and female subjects were asked to wear a sports bra. A set of markers (light-blue paper stickers of $1.25 \mathrm{~cm}$ diameter) were attached to anatomical landmarks on the subject's torso, neck, shoulders, and arms. The subject was asked to stand upright in the center of the scanner volume and assume 9 different poses with the right arm and shoulder (Fig. 1). The pose set included shoulder elevations at $-45^{\circ}$ (the arm pointing down), $0^{\circ}$ (horizontal), and $45^{\circ}$ (pointing up) in permutation with horizontal rotations at $0^{\circ}$ (pointing rightward), $45^{\circ}$, and $90^{\circ}$ (pointing forward). The forearm was extended and the hand was open in a "natural" pose without attempting to make pronation or supination. The left arm remained in a neutral pose beside the torso. Each pose was assumed upon the experimenter's instruction, and the body geometry of the corresponding pose was captured using the $3 \mathrm{dMD}$ body scanner. The subject was asked to return to a neutral pose (arms at sides) after each scan. Each scan was completed within approximately 0.8 seconds and each pose was scanned for three times. The testing procedure was approved by the NASA Internal Review Board.

A

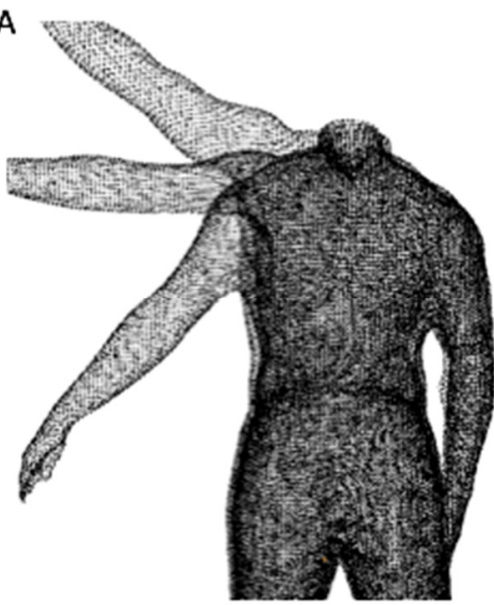

B

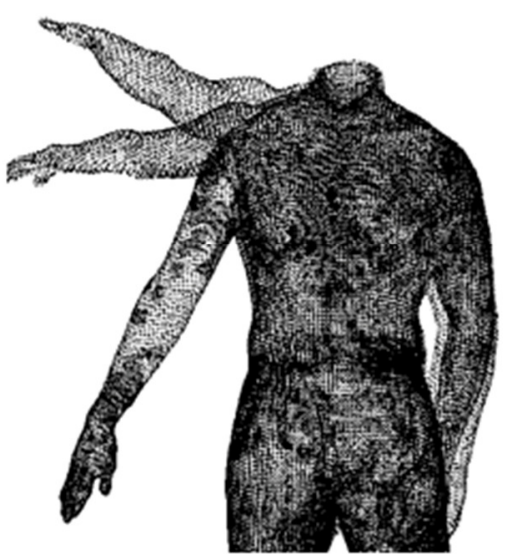

C

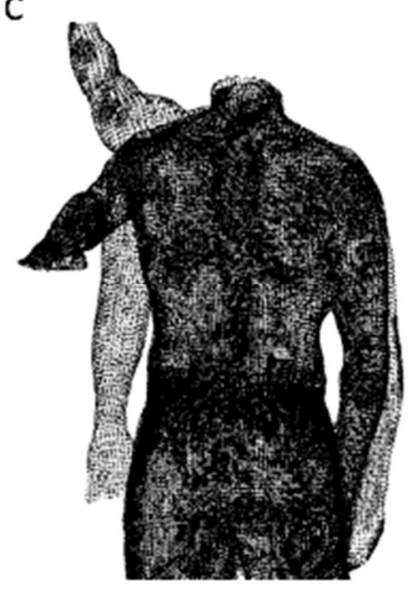

Fig. 1. Scanned poses with the right shoulder elevated to $-45^{\circ}$ (pointing down), $0^{\circ}$ and $45^{\circ}$ (pointing up). Shoulder horizontal rotation to $0^{\circ}(A), 45^{\circ}(B)$, and $90^{\circ}(C)$.

\subsection{Data Processing}

The geometry from the neck and above was removed from the raw scan data to de-identify the subjects. Each scan was hole-filled using a surface Poisson reconstruction technique [6] in MeshLab v1.3.3 software [7]. The raw scan, originally consisting in approximately 500,000 vertices, was reduced into 75,000 vertices.

The positions of the anatomical landmarks were programmatically identified on the scan data. Specifically, the color texture map associated with each scan was thresholded and filtered to isolate the pixels containing the color sticker patches placed on the skin. Then the center of each pixel cluster was located by a blob detection algorithm [8]. The identified cluster centers were manually matched and labeled with the corresponding anatomical locations.

A reference mesh ("template") was created using an open-source human modeling software MakeHuman v.1.0.2 [9]. The template, which was composed of 11,831 vertices, was used to homologically represent the scan data across different subjects and poses. In this study, the 2,043 vertices in the upper body, shoulders and extremities were taken into the modeling (Fig. 2). 
A two-step fitting process was used to align the template geometry with the scan surface. In the first step (coarse fitting), a non-rigid registration procedure ("morphing") was applied to the template. The morphing procedure was based on a radial basis function interpolation method [10], which aligns the landmark locations of the template and scanned mesh. In the second step (fine fitting), an implicit surface fitting procedure [11] moved each template vertex into the corresponding mathematical surface approximated from the scan data. The detailed steps of the fitting process have been previously described [2].

\subsection{Statistical Modeling}

The global coordinates of the upper body vertices were extracted from the fitted templates. However, global coordinates alone may not be sufficient to model the skin deformation associated with the shoulder joint articulation. Thus the local coordinates were also extracted from the right arm and shoulder segments by transforming the global coordinates of the corresponding segments into the right-arm centered coordinates (Fig. 2). A right-arm centered reference system was defined using a vector from the mid-scye (middle point between anterior and posterior scye landmarks) to the mid-elbow (middle point between the elbow tip and crease landmarks) point. A homogeneous transformation matrix was composed for transformation (Equation 1).

$$
\mathbf{p}_{i}^{\text {Local }}=\mathbf{T}_{\text {Global }}^{\text {Local }} \mathbf{p}_{i}^{\text {Global }}
$$

where $\quad \mathbf{p}_{i}^{\text {Local }}$ : The coordinate of the $i$-th vertex in the local reference frame

$\mathbf{T}_{\mathrm{Global}}^{\mathrm{Local}}$ : Homogeneous transformation matrix of the global to local reference frame

$\mathbf{p}_{i}^{\text {Global }}$ : The coordinate of the $i$-th vertex in the global reference frame

A combined data matrix was composed by concatenating the flattened global coordinates and the local coordinates, both of which correspond to the $j$-th subject and $k$-th shoulder pose (Equation 2).

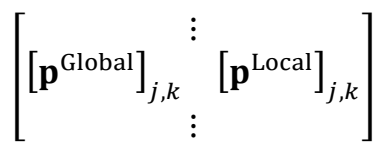

A principal component $(\mathrm{PC})$ analysis was performed on the combined data matrix. From the 108 available PC scores, the first 25 PC scores corresponding to the largest eigenvalues were selected to reduce the data dimensions for the subsequent analyses, as a preliminary analysis indicated that these 25 PC scores account for $98 \%$ of the data variance.

Each of the $25 \mathrm{PC}$ scores was predicted with a statistical linear regression (Equation 3 ) as a function of the corresponding subject's anthropometry (stature and BMI) and shoulder pose. A shoulder pose was represented by a horizontal rotation $(\theta)$ and vertical elevation $(\varphi)$ measured from the upper-extremity segment vertices similarly as for the right-arm centered reference frame.

$$
\mathrm{PC}_{m}=\boldsymbol{\beta}_{m}\left[1, \text { Stature }_{j}, \mathrm{BMI}_{j}, \theta_{j, k}, \varphi_{j, k}\right]^{\mathrm{T}}+\varepsilon
$$

$\boldsymbol{\beta}_{m}$ denotes a vector of the least-square estimates of the regression coefficients for $m$-th PC score, which minimizes the estimation error magnitudes $(\varepsilon)$.

\subsection{Parametric Geometry Estimation}

With the analysis above, the upper body geometry was estimated as a function of the anthropometry and shoulder pose parameters. Specifically, PC scores were predicted as in Equation 3, and a combined data matrix was reconstructed by an inverse transformation of the predicted PC scores. The reconstructed data matrix was separated into the global and local coordinate matrices, as defined in Equation 2.

The entire upper body, including the torso and both left and right upper extremities and shoulders, was preliminarily estimated using the global coordinates. For the right shoulder and upper extremity segments, however, an additional set of coordinates ( $\widehat{\mathbf{p}}_{i}^{\mathrm{Global}}$ ) were estimated by transforming the local coordinates (Equation 4). A homogeneous transformation matrix $\mathbf{T}_{\mathrm{Local}}^{\text {Global }}(\theta, \varphi)$ represents the global with respect to a local reference frame constructed for the given shoulder pose parameters $(\theta, \varphi)$.

$$
\widehat{\mathbf{p}}_{i}^{\text {Global }}=\mathbf{T}_{\text {Local }}^{\text {Global }}(\theta, \varphi) \mathbf{p}_{i}^{\text {Local }}
$$


Thus, the right shoulder and upper extremity geometry can be constructed either of two different sets of vertex coordinates, namely the global coordinates and the coordinates estimated from the local-to-global transformation (Equation 4). To resolve the redundancy, a weighted average function (Equation 5) was applied to determine the final averaged coordinates ( $\mathbf{p}_{i}^{\text {Averaged }}$ ).

$$
\mathbf{p}_{i}^{\text {Averaged }}=\left(1-w_{i}\right) \mathbf{p}_{i}^{\text {Global }}+w_{i} \widehat{\mathbf{p}}_{i}^{\text {Global }}
$$

The weight coefficients $\left(w_{i}\right)$ were assigned on the template in a way that the value at the mid-scye regions corresponds to 0.5 and gradually increases to 1 as the vertex is located more adjacent to the elbow, and decreases to 0 in the opposite direction toward the torso (Fig. 2).

The final upper body geometry was constructed using the weight-averaged vertex coordinates. The estimation accuracy and errors were also assessed by the $L^{2}$-norm between the measured and estimated vertex coordinates.

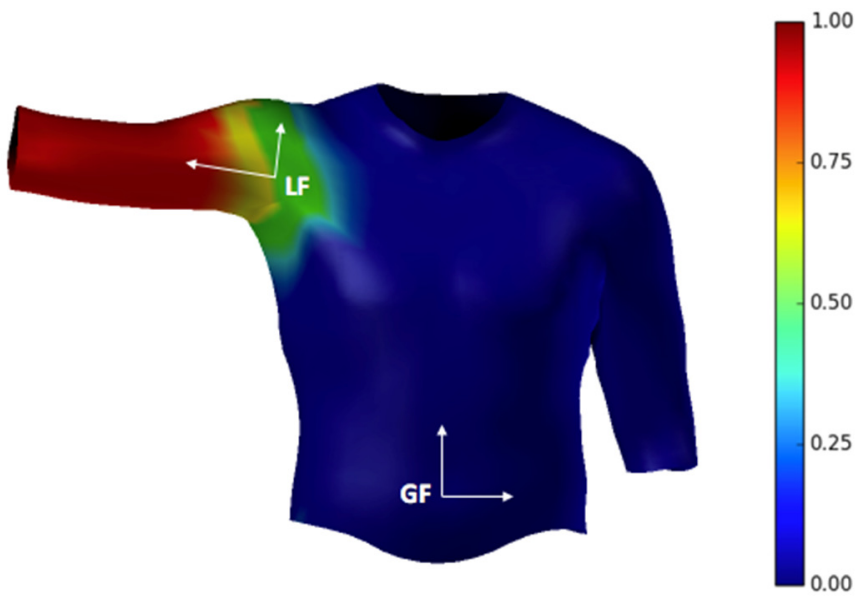

Fig. 2. Upper body template. GF: Global reference frame. LF: Shoulder-centered local reference frame. The dark blue versus red area indicates a higher weight assigned to the global versus shoulder-centered local coordinates for geometry estimation, respectively.

\section{Results}

\subsection{Model-Estimated Geometry}

A set of sample body shapes generated by the model are illustrated in Fig. 3. The model parameters corresponded to a large $\left(190 \mathrm{~cm}\right.$ stature and $\left.30 \mathrm{~kg} / \mathrm{m}^{2} \mathrm{BMI}\right)$, medium $\left(175 \mathrm{~cm}\right.$ stature and $25 \mathrm{~kg} / \mathrm{m}^{2}$ BMI), and small body size $\left(160 \mathrm{~cm}\right.$ stature and $\left.20 \mathrm{~kg} / \mathrm{m}^{2} \mathrm{BMI}\right)$. The shoulder poses were also varied across multiple different combinations of horizontal rotations and vertical elevations. These arm poses are representative of motions and ranges performed while wearing a space suit [12].
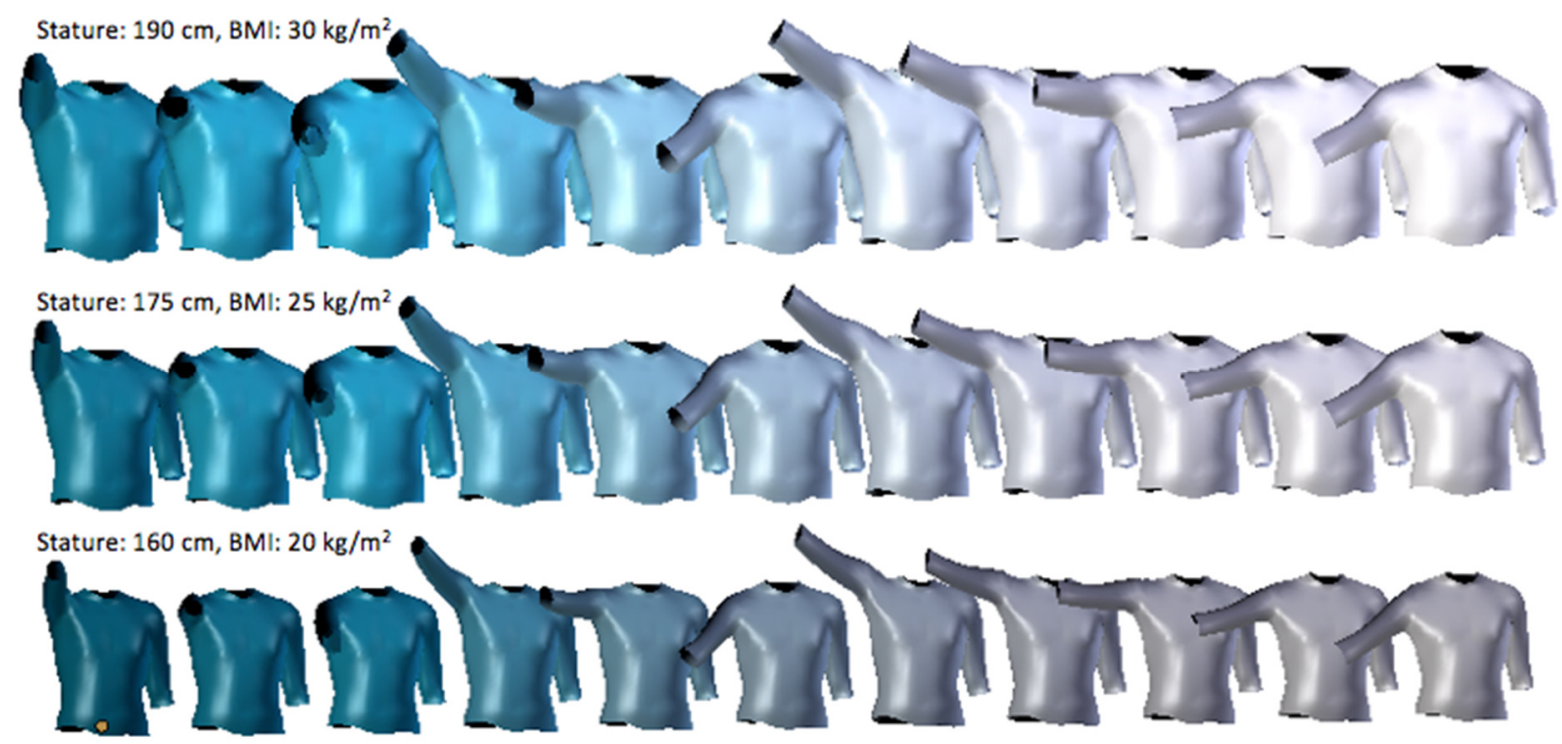

Fig 3. Body shapes generated by the model using different anthropometry and shoulder pose parameters. 


\subsection{Estimation Accuracy}

For model accuracy assessments, body shapes were first generated using the stature and BMI measurements of each scanned subject. Then the model errors were calculated by calculating the $L^{2}$-norms between the corresponding subject scan versus model-estimated vertex coordinates. The errors were averaged across all the subjects. Two sample shoulder poses were considered (Fig. 4). The errors are 12.90 (SD 6.88) $\mathrm{mm}$ on average for the shoulder pose with the right arm rotated forward to $45^{\circ}$ and lowered by $45^{\circ}$ (Fig. 4A). Similarly, the errors are 11.98 (SD 6.59) $\mathrm{mm}$ for the rotated forward by $45^{\circ}$ and elevated up by $45^{\circ}$ (Fig. 4B). Overall the errors tend to be larger in the arms at the biceps and triceps areas and upper abdominal areas.
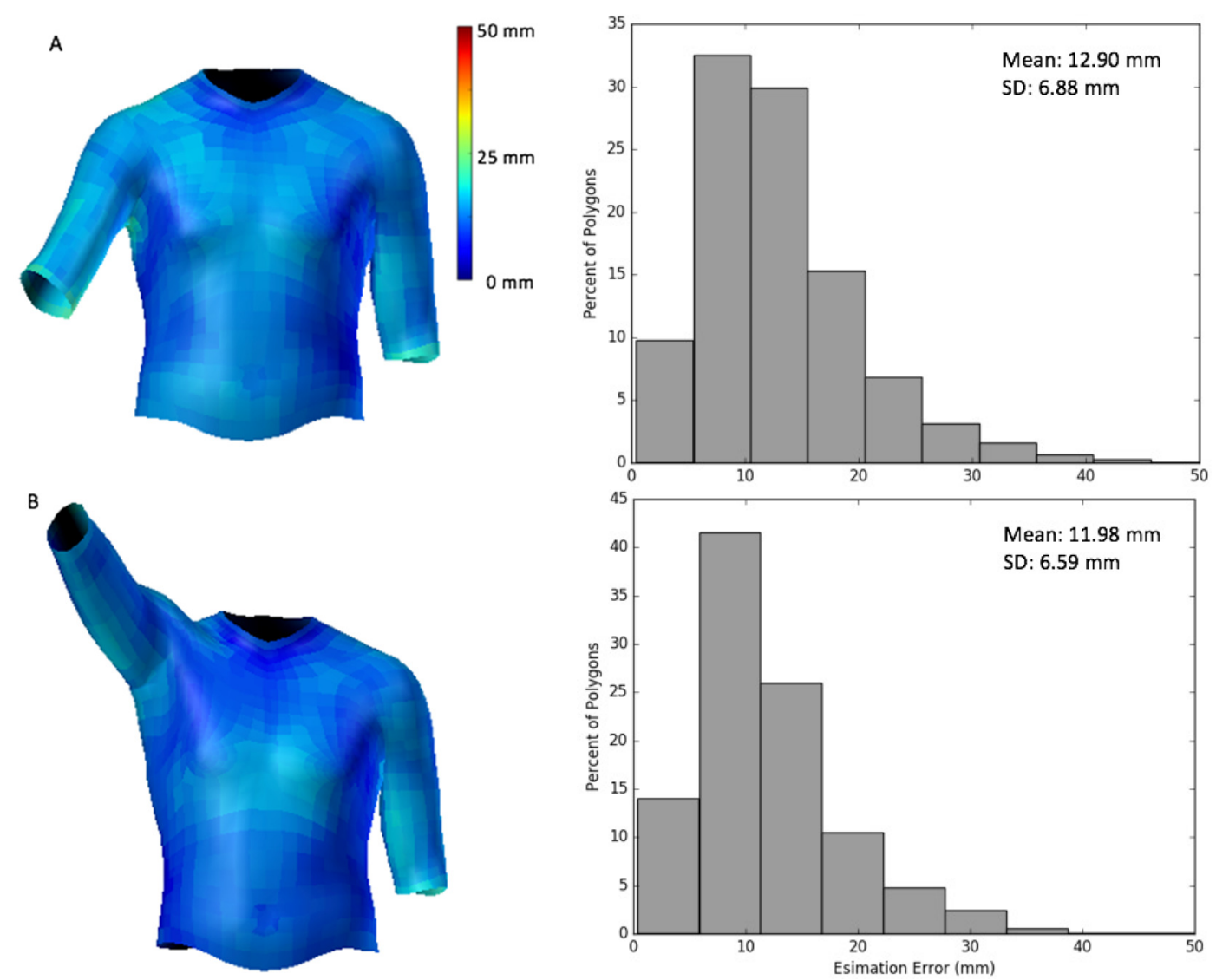

Fig. 4. Model estimation errors for sample poses.

\subsection{Application to Suit Fit Assessments}

The model-estimated body shapes were incorporated with the CAD drawings of a space suit (Fig. 5), specifically a medium-size Extravehicular Mobility Unit (EMU), including the hard upper torso (HUT), display and control module (DCM), scye rings, and upper arm assembly excluding soft goods. The sample body shapes were generated using a parameter set of $176 \mathrm{~cm}$ stature, $25 \mathrm{~kg} / \mathrm{m}^{2}$ BMl. The shoulder poses corresponded to $30^{\circ}$ horizontal rotation forward and $-20^{\circ}$ (Fig. 5A) and $20^{\circ}$ (Fig. 5B) vertical elevations. The position of the body shape and EMU joint configurations were manually adjusted in a way to minimize the overlaps.

Overall, the sample illustrations demonstrate that the model-estimated body shapes and space suit $\mathrm{CAD}$ drawings can be incorporated to quantify the contact volume and clearance between the suit and body surfaces. For example, the clearance on the rear of the upper arm reduces as the shoulder is in an elevated pose, as shown in Fig. 5B. In general, it was observed that the clearance tends to be reduced as the shoulder approaches extreme positions, such as $0^{\circ}$ and $90^{\circ}$ horizontal rotations and $30^{\circ}$ and $-30^{\circ}$ vertical elevations. 

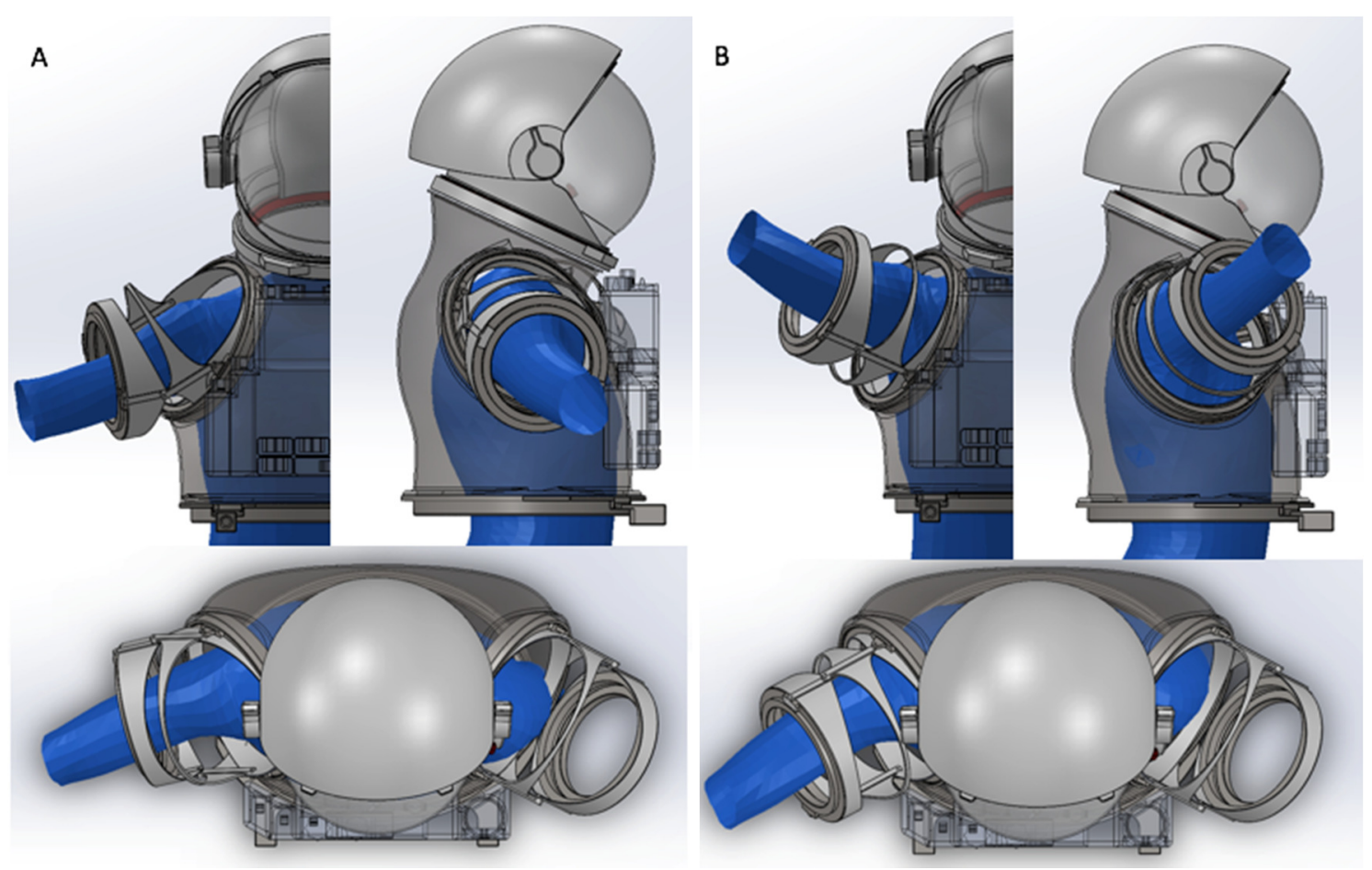

Fig. 5. Model estimated body geometry incorporated with the CAD drawings of an Extravehicular Mobility Unit (EMU) space suit.

\section{Discussion}

In this study, a model framework was developed to parametrically estimate an upper body geometry as a function of the anthropometry of the target population and shoulder poses of interest. Based on the scans of nine different shoulder poses, a two-step fitting technique, namely an initial morphing followed by an implicit surface fitting, generated a homological representation of body shapes across the scans in different body sizes and poses. A statistical model of the global and local coordinates of the fitted templates was able to reconstruct the skin deformation patterns varying with the different articulations at the shoulder. The estimation errors are approximately $11-12 \mathrm{~mm}$ on average, which are suitable for the purpose of this study to assess space suit. The fit assessment in a CAD environment was demonstrated as a proof of concept. Future effort will be made to geometrically quantify and analyze the interactions between the suit and body surface.

This model is based on the upper body poses articulated without wearing a suit ("active poses"). However, it has been reported that wearing a space suit, particularly with the HUT and scye bearings, generally changes the patterns of upper-body poses [12], [13]. For example, the orientation of the scye bearings often results in subjects protracting their shoulders forward to a much greater extent even in a neutral pose than they would normally do when standing neutrally unsuited. Thus the resulting poses in a suit may not be an exact match to the active poses considered in this study. A future study should represent the "passive" poses that suit geometry forces the body into.

Another limitation of the present model is the combination of male and female scan data due to the insufficient number of subjects. With more subjects across different body shapes, other crucial factors for the skin morphology can be more accurately represented, including gender and muscularity. Further the present model used a weighted averaging map pre-defined on the template as illustrated in Fig. 2. This approach was simple to implement and demonstrated a good level of estimation accuracy. However, future effort can be made to statistically survey and analyze the weight mapping patterns directly from the scan data.

Overall, the model developed in this study can be used to predict the skin deformation and shape variations for any body size and shoulder pose for the crewmember population. It is expected that the virtual fit process that can be developed from this study would help to reduce the time and cost of the iterative processes for the suit design, fit and evaluation. 


\section{References}

[1] D. R. Williams and B. J. Johnson, "EMU Shoulder Injury Tiger Team Report," National Aeronautics and Space Administration, NASA/TM-2003-212058, S-912, Sep. 2003.

[2] B.-K. Park and M. P. Reed, "Parametric body shape model of standing children aged 3-11 years," Ergonomics, vol. 58, no. 10, pp. 1714-1725, Oct. 2015.

[3] N. Vaughan, V. N. Dubey, M. Y. K. Wee, and R. Isaacs, "Parametric model of human body shape and ligaments for patient-specific epidural simulation," Artif. Intell. Med., vol. 62, no. 2, pp. 129140, Oct. 2014.

[4] A. Nerot, W. Skalli, and X. Wang, "A principal component analysis of the relationship between the external body shape and internal skeleton for the upper body," J. Biomech.

[5] S. Margerum, M. Ferrer, K. Young, and S. Rajulu, "Relating Linear and Volumetric Variables through Body Scanning to Improve Human Interfaces in Space," in Proceedings of the First International Conference on 3D Body Scanning Technologies, Lugano, Switzerland, 2010, pp. 010-022.

[6] M. Kazhdan, M. Bolitho, and H. Hoppe, "Poisson Surface Reconstruction," in Proceedings of the Fourth Eurographics Symposium on Geometry Processing, Aire-la-Ville, Switzerland, Switzerland, 2006, pp. 61-70.

[7] "MeshLab." [Online]. Available: http://meshlab.sourceforge.net/.

[8] "Common Interfaces of Feature Detectors - OpenCV 2.4.13.1 documentation." [Online]. Available: http://docs.opencv.org/2.4/.

[9] "MakeHuman | Open source tool for making 3d characters." [Online]. Available: http://www.makehuman.org/.

[10]H. E. Bennink, J. M. Korbeeck, B. J. Janssen, and B. M. ter H. Romeny, "Warping a Neuro-Anatomy Atlas on 3D MRI Data with Radial Basis Functions," in 3rd Kuala Lumpur International Conference on Biomedical Engineering 2006, F. Ibrahim, N. A. A. Osman, J. Usman, and N. A. Kadri, Eds. Springer Berlin Heidelberg, 2007, pp. 28-32.

[11]J. C. Carr et al., "Reconstruction and Representation of 3D Objects with Radial Basis Functions," in Proceedings of the 28th Annual Conference on Computer Graphics and Interactive Techniques, New York, NY, USA, 2001, pp. 67-76.

[12] C. R. Reid et al., "An Ergonomic Evaluation of the Extravehicular Mobility Unit (EMU) Spacesuit Hard Upper Torso (HUT) Size Effect on Mobility, Strength, and Metabolic Performance," Proc. Hum. Factors Ergon. Soc. Annu. Meet., vol. 58, no. 1, pp. 1595-1599, Sep. 2014.

[13] R. Z. Amick, C. R. Reid, S. A. England, and S. L. Rajulu, "Characterization of Joint Resistance and Performance Degradation of the Extravehicular Mobility Unit Spacesuit A Pilot Study," Proc. Hum. Factors Ergon. Soc. Annu. Meet., vol. 59, no. 1, pp. 1259-1263, Sep. 2015. 